\title{
Cryptocurrency platform ecosystem: a systematic literature review from information systems perspective
}

\author{
Shehu M. Sarkintudu ${ }^{1 *}$, Huda H. Ibrahim ${ }^{2}$ and Alawiyah Abd Wahab ${ }^{3}$ \\ $\mathrm{PhD}$ Candidate, Institute for Advanced and Smart Digital Opportunities, School of Computing, Universiti Utara \\ Malaysia 06010 Sintok, Kedah, Malaysia ${ }^{1}$ \\ Professor, Institute for Advanced and Smart Digital Opportunities, School of Computing, Universiti Utara Malaysia \\ 06010 Sintok, Kedah, Malaysia ${ }^{2}$ \\ Senior Lecturer, Institute for Advanced and Smart Digital Opportunities, School of Computing, Universiti Utara \\ Malaysia 06010 Sintok, Kedah, Malaysia ${ }^{3}$
}

Received: 24-September-2018; Revised: 20-December-2018; Accepted: 30-January-2019

(C)2019 Shehu M. Sarkintudu et al. This is an open access article distributed under the Creative Commons Attribution (CC BY) License, which permits unrestricted use, distribution, and reproduction in any medium, provided the original work is properly cited.

\begin{abstract}
Cryptocurrency platform continues to attract public attention in recent times as an alternative payment platform. The platform forms a new ecosystem of different entities such as users, cryptocurrency exchange, wallet providers, miners and platform developers that are interacting together in a value. Information systems (IS) and other related fields' scholars continue to explore the evolution of these emerging disruptive financial innovations. However, there is a lack of systematic overview that focus on the cryptocurrency platform ecosystem layer. Thus, the objectives of this work are: (1) to identify the number of published IS and other related fields researches that contributed to the ecosystem layer; (2) to determine the research methods and theories used in these published works; and (3) to suggest potential research opportunities in cryptocurrency. A systematic review following [1] and [2] guidelines was conducted to address these objectives. The review analyzes the existing literature published between the years of 2012 to 2017. This period was chosen because cryptocurrency phenomenon is a new field. The results of the review show that there is an inadequate utilization of IS theories and methods in reporting the previous findings. This paper presents and compares the contributions of IS and other IS related fields, scholars regarding the cryptocurrency ecosystem evolution. Finally, the paper concludes by discussing the implications for researchers and practitioners and outlining the potential open research opportunities.
\end{abstract}

\section{Keywords}

Cryptocurrency, Digital payment, Bitcoin platform, Disruptive technology, Platform ecosystems.

\section{Introduction}

Cryptocurrency exhibits core properties of digital information system (IS) artifact that provides a new innovative platform that has the capability to store, send and receive electronic money [3]. In 2008, the first cryptocurrency platform project was released in a white paper by an anonymous named Satoshi Nakamoto [4]. Today, as of September 2018, Bitcoin is the most widely used cryptocurrency with a market capitalization of 110 billion USD [5]. As a result of global financial crisis in 2007, many people began to lose confidence in the centralized financial platform and start to look for a viable decentralized currency platform as an option [6].

*Author for correspondence 308
Consequently, this led to the emergence of the cryptocurrency platform. The platform continues to draw global attention as an alternative currency and payment system, but remain somewhat difficult to understand by the larger society [7]. Such platform is considered as a disruptive technology with a phenomenon that holds a huge potential for multifaceted research that cut across technology and societal context [8].

A number of previous articles obtained from the IS research databases and conferences on cryptocurrency platform emphasize on protocol and network layers which are more of the technical perspective. However, there are very few articles that focused on the practitioners as they can be a source of knowledge on the ecosystem layer of the platform 
[9]. Furthermore, most of these reviews are limited to only a summary of cryptocurrency and alignment to IS research. However, it is necessary to include more databases, journals and conferences from IS related disciplines such as Economics and Law that may further enhance the insight on the cryptocurrency platform particularly on the ecosystem layer [8].

Recent research [10] has found that, theoretically, the platform is free and available to anyone who has access to a computer and internet connection. The platform also represents yet another potentially disruptive internet technology, leading to the fundamental question of whether it is truly free and easily available to use. Since the introduction of bitcoin by Satoshi [4], some crypto-currency software such as the bitcoin core reference client remains a free and open source. However, the cryptocurrency platform remains as a complex technology that requires the help of intermediaries such as complementary products and service providers. This open, innovative platform solves the problem of how two or more people who are anonymous to each other can establish trust and mutually agree upon a transaction without a central entity. The public ledger otherwise known as blockchain is transparent and freely downloadable. Cryptocurrency is gradually gaining research attention not only in IS and computer science, but also in other disciplines such as Economics and Law. This shows the link between cryptocurrency to various interrelated domains.

Cryptocurrency research in IS can be considered as a relatively young research field. The review of this emerging startup ecosystem may become a starting point for researchers to understand potential research opportunities. The main focus of this systematic review paper is on the ecosystem layer of the cryptocurrency, which requires a critical understanding of the ecosystem layer issues since related research in the field is still scanty within the IS and its related research communities.

\section{Platform}

Technological platform is a valuable network system that mediates two or more multi-sided entities [11]. Cryptocurrency is a blockchain-enabled distributed platform that is similar to any other contemporary multi-sided platforms that employ split revenue, introduce new models of platform governance, have product and services which have been distributed across various market sides in a more equilibrium manner without monopoly [12].
Similarly, the distributed and traditional platforms coordinate, regulate and motivate platform developers to foster networking effects as much as what has been done by the conventional multi-sided platform owners in order to maximize their own benefits. However, the only difference is that there is no tool to exert monopoly in the distributed platforms [13].

Mattila and Seppala [12] found that the structures of the crypto-currency distributed platform have not yet been thoroughly explored in the context of multisided platform literature. However, according to Moore and Christin [14] cryptocurrency is a platform for illegal activities such as money laundering and selling illicit goods that might stimulate further usage and activate new users in the future. On the other hand, the platform is applauded for its low transaction fees and promoted as a viable alternative to banks and credit cards [15].

Most cryptocurrencies such as etheurium and litecoin ripple share the same fundamental ideas with the initial Bitcoin [16]. Since the emergence of the platform, researchers on cryptocurrency pay more attention on the protocol and network layers with less emphasis on the ecosystem. As for the IS scholars, this requires a more vivid discussion [8]. Therefore, the main focus of this review is on the behavioral perspective of the ecosystem layer [17].

\section{Ecosystem layer}

An ecosystem is defined as a network of entities or stakeholders that are gathered or interacting around a single platform, called Software ecosystems [10, 18, 19]. A cryprotocurrency platform ecosystem is a technology such as Windows, iOS, or Android ecosystem, which is in its early stage that brings multiple parties together for a common purpose to perform financial transactions over the Internet.

While the traditional technology ecosystem shares profit between external complementary asset providers and platform's owner, the cryptocurrency platform ecosystem splits its revenue among all various market sides that participate in the platform provision and the ecosystem at large. The growing numbers of cryptocurrency users and global media attention have raised the interest of various research communities on the platform. Since research on crypto-currency has many segments, it is likely to continue to drag the attention of the research effort of the IS special interest groups such as e-business and information security [8]. 
Previous research effort in cryptocurrency ecosystem layer shows that the platform is vulnerable to money laundering and illegal transactions [20, 21]. For example, Moore and Christin [14] described the use of Bitcoin to purchase prohibited items such as narcotics and other harmful drugs. It is also found that even though they are still very few, the core platform developers have a high influence on the protocol maintenance and management as compared to the large portion of participants in the ecosystem who have limited influence [8]. This led to the coordination and security issues.

Another central issue is about mining a new currency into the network. Due to high electricity consumption and cost of mining equipment [22], many miners have been pushed to form mining pools and jointly contribute to computational power and share the reward from their proof of work as it obtains in the Bitcoin eco-system [23]. This development may lead to many collections of pools that will likely to collude and acquire more than 50 percent of computing power share. Having such plot, they could effectively control the network and prevent certain valid transactions or approve double spending in the ecosystem [24].

Although the cryptocurrency platform is also a topic in Computer Science, Economics and Law, this study only looks into the contributions of the IS related fields to the ecosystem platform layers and compare it to those of the IS field. The goal of this study is to provide an overview of previous research that focused on the cryptocurrency platform ecosystem layer. The followings are the research questions:

RQ1: How many published IS and related fields contribute to the cryptocurrency platform ecosystem layer?

RQ2: How many research methods and theories that have been used in studying the cryptocurrency platform ecosystem layer?

RQ3: What are the potential research opportunities on the cryptocurrency platform ecosystem layer?

\section{Methodology}

This study followed the guidelines for conducting a systematic literature review provided by $[1,2]$.
Firstly, the review process started by developing a simple protocol that outlines the research questions, the search strategy, the selection criteria, the quality assessment criteria and the data extraction strategy. The following sections summarize the conducted review process.

\subsection{The search process}

In order to find the relevant articles to be included in the review, a systematic search for cryptocurrencies literature from the point of view of the IS domain was conducted [25]. The leading databases in IS such as AIS Electronic Library, Scopus, and Web of Science were investigated. The specific search terms used were "digital currency", "crypto", "cryptocurrency", "Bitcoin", "Blockchain" "distributed ledger" and "Bitcoin platform ecosystem" which resulted to 150 primary sources in addition to another two (2) review articles. Thus, the total number of articles was 152 .

\subsection{The selection process}

At this stage, the articles were selected based on these three criteria: 1) AIS senior scholars journals from 2012 to 2017 such as AJIS, CAIS, EJIS, ISJ, JAIS JISTEM, JMAIS, JMWAIS, PAJAIS, 2) higher ranked IS chapters conference proceedings such as AMCIS, ECIS, HICSS, ICIS, MCIS, PACIS, and 3) other related and non-IS databases such as EBSCohost, IEEE, ACM. From the selection, only five articles fulfilled the first criteria whilst 73 and 74 met the second and third respectively as shown in Figure 1. The process began by removing doubles and comparing the titles and abstracts of all the search results as well as controlling the number of citations of the individual articles.

To ensure that only relevant articles were selected, systematic forward and backward citations, publications impact factors comparison, and full texts scrutiny were carried out. The selected samples were cut down to 42 that represent the highly relevant articles. This was done by ensuring that at least one of the search terms appears in either at the title, keyword or abstract of the journal or conference articles. Articles that did not meet this requirement were excluded in the next round of review. However, for the non-IS journal and conference articles, only those that focused on the cryptocurrency's platform ecosystem were considered for further review. Table 1 shows the detail sources of articles drawn at each stage of the selection process. 


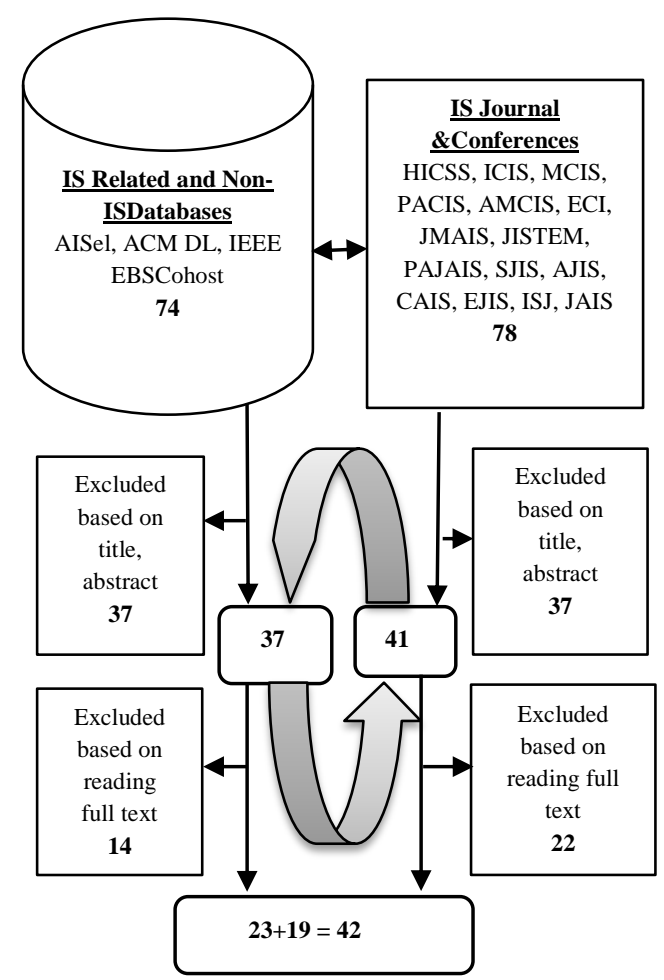

Figure 1 Search and selection procedure

Based on Table 1, 20 and 22 papers were selected from IS outlet and non-IS outlet respectively. Of 20 papers from the IS outlet, two (2) papers were selected from IS journal, 17 papers were selected from IS conferences while only one (1) paper was selected from IS related databases. Of 22 papers from non-IS outlet, 15 papers were selected from Computer Science area, five (5) from Economics and two (2) from Law.

\subsection{Quality assessment}

The quality of the selected articles for the review was assessed based on whether the study presented clear objectives, provided appropriate data collection method, discussed appropriate data analysis method, and presented clearly stated findings. For example, if the objectives were clear, a score of 1 is given, if the objectives were partly clear, a score of 0.5 is given and if the objectives were not clear, a score of 0 is given. The quality assessment criteria were adapted from [26]. The total score for each article is calculated by adding the score. Table 2 shows the results of the quality assessment. Of 42 papers, 22 papers were rated very well and only three (3) papers were rated poor.

\subsection{Data extraction}

The reference management software, Mendeley software platform (http://www.mendeley.com/) was used to record the reference details for each article. The data that was extracted to answer each of the research questions includes the disciplines of study, the methods and the theory used.

Table 1 Selection result

\begin{tabular}{|c|c|c|c|c|c|c|}
\hline & & Sources & & Search articles & $\begin{array}{l}\text { Selection based } \\
\text { on titles and } \\
\text { abstract }\end{array}$ & $\begin{array}{l}\text { Selection } \\
\text { based on } \\
\text { full text }\end{array}$ \\
\hline \multirow{17}{*}{ 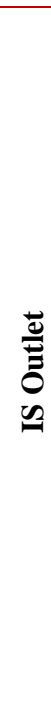 } & \multirow{10}{*}{$\begin{array}{l}\frac{n}{\tilde{E}} \\
\stackrel{\Xi}{\Xi} \\
\stackrel{0}{\mathscr{n}}\end{array}$} & \multirow{10}{*}{$\underset{\Xi}{\overparen{\Xi}}$} & JMWAIS & 0 & 0 & 0 \\
\hline & & & PAJAIS & 1 & 0 & 0 \\
\hline & & & SJIS & 0 & 0 & 0 \\
\hline & & & AJIS & 0 & 0 & 0 \\
\hline & & & CAIS & 1 & 0 & 0 \\
\hline & & & EJIS & 1 & 1 & 1 \\
\hline & & & ISJ & 1 & 0 & 0 \\
\hline & & & JAIS & 0 & 0 & 0 \\
\hline & & & JISTEM & 1 & 1 & 1 \\
\hline & & & JMAIS & 0 & 0 & 0 \\
\hline & \multirow{7}{*}{ 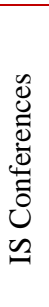 } & & HICSS & 7 & 2 & 0 \\
\hline & & & ICIS & 16 & 10 & 5 \\
\hline & & & MCIS & 12 & 2 & 1 \\
\hline & & & PACIS & 3 & 2 & 1 \\
\hline & & & AMCIS & 12 & 4 & 3 \\
\hline & & & ECIS & 21 & 17 & 5 \\
\hline & & & IS (SLR) & 2 & 2 & 2 \\
\hline
\end{tabular}




\begin{tabular}{|c|c|c|c|c|c|}
\hline & \multirow{3}{*}{ 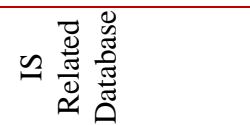 } & IEEE & 9 & 1 & 1 \\
\hline & & Ebcohost & 2 & 0 & 0 \\
\hline & & ACM & 11 & 0 & 0 \\
\hline & Computer Science & & 38 & 28 & 15 \\
\hline$\frac{2}{1}$ & Economics & & 12 & 6 & 5 \\
\hline อี & Law & & 2 & 2 & 2 \\
\hline & & & 152 & 78 & 42 \\
\hline
\end{tabular}

Table 2 Quality score

\begin{tabular}{lccccc}
\hline Scale & \multicolumn{2}{c}{ Quality (scores) } & & Total \\
\hline & $\begin{array}{c}\text { Poor } \\
(\mathbf{1 - 1 . 9 9 )}\end{array}$ & $\begin{array}{c}\text { Fair } \\
(\mathbf{2 - 2 . 9 9 )}\end{array}$ & $\begin{array}{c}\text { Good } \\
\mathbf{( 3 - 3 . 9 9 )}\end{array}$ & $\begin{array}{c}\text { VGood } \\
\text { (4) }\end{array}$ & \\
\hline Number of studies & 3 & 6 & 11 & 22 & 42 \\
\hline $\begin{array}{c}\text { Percentage of papers } \\
(\%)\end{array}$ & 7.1 & 14.3 & 26.2 & 52.4 & 100 \\
\hline
\end{tabular}

\section{Findings}

\subsection{Point of views}

The findings of this review were extracted according to the point of views of various disciplines, theories, research methods, the investigated issues and future research directions based on the comparative analysis of the current trend in cryptocurrency platform ecosystem research field. The perspectives of various research fields are shown in Table 3.

Table 3 Point of view

\begin{tabular}{lc}
\hline Perspectives & Number of papers \\
\hline Information Systems & 13 \\
Computer Science & 22 \\
Economics & 4 \\
Law & 2 \\
\hline
\end{tabular}

\subsection{Theories used}

To answer research question two (2), the theories used in the articles were classified into IS, Non-IS and Not-Available as listed below (Table 4).

Table 4 Theories

\begin{tabular}{lc}
\hline Articles based on theories & No. of articles \\
\hline IS theories used & 5 \\
\hline Non-IS theories used & 4 \\
\hline Not Available & 33
\end{tabular}

The findings reveal that only five (5) out of the 42 articles that represent the IS contributions on cryptocurrencies platform eco-system used IS theories. Connolly and Kick [27] used diffusion of innovation theory [28] to understand how cryptocurrencies platform is adopted over time. In addition, Polasik et al. [29] used the same theory to understand the price fluctuation and use of bitcoin in its ecosystem. Walton and Dhillon [30] used Game Theory, another promising IS theory, and combined it with the bounded rationality theory to understand digital crime and trust in the blockchain platform. The combination of these theories enables the scholars to access the trust and crime issues in bitcoin platform ecosystem. However, to the best of our knowledge, only four (4) non-IS theories were used to study the cryptocurrency platform ecosystem. For example, the Rational Choice Theory was used by Janze and Gvozdevskiy [31] to examine the coevolution of the bitcoin platform, whilst Rukanova et al. [32] used the Collective Action Theory to understand the collective innovation of the distributed and decentralized environment. As for Glaser [33], the Descriptive Theory was applied to study the decentralized platform.

The evidence from this review shows that IS scholars still have not done much in reporting the scientific evidence of cryptocurrencies platform ecosystem due to the limitation on the use of IS theories. Hence, this opportunity needs to be explored.

\subsection{Methods used}

Based on the results of extensive reading and rereading of the IS articles, the research methods used by the authors were classified into nine (9) categories as depicted in Table 5. Among the categories include Qualitative, Quantitative, Literature Review, Not Available and Mixed. 
Table 5 Research methods

\begin{tabular}{ll}
\hline Research methods & No of articles used \\
\hline Qualitative & 6 \\
\hline Quantitative & 11 \\
\hline Mixed methods & 2 \\
\hline Literature review & 2 \\
\hline Design science & 1 \\
\hline Prototype & 1 \\
\hline Case study & 3 \\
\hline Heuristic clustering & 2 \\
\hline Not available & 14 \\
\hline
\end{tabular}

The findings indicate that majority of the articles that have not used or presented any research methods were considered as Not Available category [34, 35]. Since the field is relatively new, most publications merely present the introduction of the crytocurrency ecosystem [21, 29, 36-38] and lack scientific evidence in their findings.

\section{Potential research opportunities on cryptocurrency platform ecosystem layer}

The studies presented so far provide an insight that can be useful to the IS research communities and practitioners. Literature that focused on examining the cryptocurrency ecosystem are merely simple introductory to cryptocurrency. For example, papers by $[10,19,37,38]$ provide an insight into the cryptocurrency ecosystem perspective. It is also found that many of these papers lack the step-by-step procedures of conducting a research. Although some scholars present their scientific findings using certain methodology, more need to be done by the IS scholars in applying existing theories and methods to understand this promising domain.

This study, so far, has provided significant evidence that might be useful for both the industry players and academic communities, especially the IS scholars, to look into areas of cryptocurrencies platform ecosystem that are worth to be explored in the future. The findings also indicate that both the IS and IS related point of views focused on introducing and explaining the performance by emphasizing on the adoption and prediction of the cryptocurrency platform rather than explaining the interaction between the core platform and its sub-modules independently without compromising the core platform over time due to openness.
Furthermore, this study confirmed that very little attention has been paid in linking the IS theories in understanding the evolution of the platform ecosystem that may contribute to further theory development.

\section{Conclusion}

In spite of many research efforts by both the IS and other IS related fields in understanding the cryptocurrency platform ecosystem as a disruptive technology, there are still promising possibilities of enhancing the understanding by looking into its progress and influence particularly on the matters concerning the modern financial process and procedure. This study has confirmed the need for more investigations on the interaction of the entities within the cryptocurrency platform and the formation of the emerging eco-system.

Even though the cryptocurrency platform ecosystem is a young and growing field of research, not much effort has been made to link the fundamental existing theories in supporting the findings. In addition, the use of scientific methods to provide rigorous output in understanding the emerging phenomenon is still lacking. Therefore, the conclusion cannot be recommended without acknowledging the limitations of this study. First and foremost, in conducting this study, the data gathered do not include the Business and Sociology journals that also investigate issues on cryptocurrency.

Compared to other related systematic literature review studies conducted previously, this study has moved forward by not just limiting the inclusion of IS outlets data. Furthermore, the study focuses on the bitcoin as the most used cryptocurrency platform ecosystem, even though there are other emerging alternative platforms. The other platforms will be considered as future work. 


\section{Acknowledgment}

The work presented in this paper was partly supported by the Ministry of Education Malaysia under the Research Acculturation Collaborative Effort (RACE) grant (S/O code: 12962).

\section{Conflicts of interest}

The authors have no conflicts of interest to declare.

\section{References}

[1] Keele S. Guidelines for performing systematic literature reviews in software engineering. Technical report, Ver. 2.3 EBSE Technical Report. EBSE; 2007.

[2] Webster J, Watson RT. Analyzing the past to prepare for the future: writing a literature review. MIS Quarterly. 2002; 26(2):13-23.

[3] Kazan E, Tan CW, Lim ET. Value creation in cryptocurrency networks: towards a taxonomy of digital business models for bitcoin companies. In PACIS 2015.

[4] Nakamoto S. Bitcoin: a peer-to-peer electronic cash system. 2008 .

[5] https://coinmarketcap.com/all/views/all/. Accessed 26 October 2018.

[6] Teigland R, Yetis Z, Larsson T. Breaking out of the bank in Europe-exploring collective emergent institutional entrepreneurship through bitcoin. SSRN. 2013.

[7] Van Alstyne M. The magazine archive includes every article published in communications of the ACM for over the past 50 years. Communications of the ACM. 2014; 57(5):30-2.

[8] Morisse M. Cryptocurrencies and bitcoin: charting the research landscape.2015.

[9] Oshodin O, Molla A, Ong CE. An information systems perspective on digital currencies: a systematic literature review. ACIS 2016.

[10] Cusumano MA. The bitcoin ecosystem. Communications of the ACM. 2014; 57(10):22-4.

[11] Han E, Suh B, Shin SK. Developing a reference model for analyzing mobile platform business: from an ecosystem view. AMCIS 2016.

[12] Mattila J, Seppälä T. Distributed governance in multisided platforms: a conceptual framework from case: bitcoin. In collaborative value co-creation in the platform economy 2018 (pp. 183-205). Springer, Singapore.

[13] Boudreau KJ, Hagiu A. Platform rules: multi-sided platforms as regulators. Platforms, Markets and Innovation. 2009; 1:163-91.

[14] Moore T, Christin N. Beware the middleman: empirical analysis of bitcoin-exchange risk. In international conference on financial cryptography and data security 2013 (pp. 25-33). Springer, Berlin, Heidelberg.

[15] Brito J, Castillo A. Bitcoin: a primer for policymakers. Mercatus Center at George Mason University; 2013.

[16] Bentov I, Lee C, Mizrahi A, Rosenfeld M. Proof of activity: extending bitcoin's proof of work via proof of stake. IACR Cryptology. 2014:1-19.
[17] Pant V, Yu E. Modeling simultaneous cooperation and competition among enterprises. Business \& Information Systems Engineering. 2018; 60(1):39-54.

[18] Van Den Berk I, Jansen S, Luinenburg L. Software ecosystems: a software ecosystem strategy assessment model. In proceedings of the fourth European conference on software architecture: companion 2010 (pp. 127-34). ACM.

[19] Evans PC, Gawer A. The rise of the platform enterprise: a global survey. 2016. SRI.

[20] Meiklejohn S, Pomarole M, Jordan G, Levchenko K, McCoy D, Voelker GM, et al. A fistful of bitcoins: characterizing payments among men with no names. In proceedings of the conference on internet measurement conference 2013 (pp. 127-40). ACM.

[21] Möser M, Böhme R, Breuker D. An inquiry into money laundering tools in the bitcoin ecosystem. In APWG ecrime researchers summit 2013 (pp. 1-14). IEEE.

[22] Kroll JA, Davey IC, Felten EW. The economics of bitcoin mining, or bitcoin in the presence of adversaries. In proceedings of WEIS 2013 (pp. 1-21).

[23] Lewenberg Y, Bachrach Y, Sompolinsky Y, Zohar A, Rosenschein JS. Bitcoin mining pools: a cooperative game theoretic analysis. In proceedings of the international conference on autonomous agents and multiagent systems 2015 (pp. 919-27). International Foundation for Autonomous Agents and Multiagent Systems.

[24] Gervais A, Karame GO, Capkun V, Capkun S. Is bitcoin a decentralized currency? IEEE Security \& Privacy. 2014; 12(3):54-60.

[25] Schwartz RB, Russo MC. How to quickly find articles in the top IS journals. Communications of the ACM. 2004; 47(2):98-101.

[26] Dybå T, Dingsøyr T. Empirical studies of agile software development: a systematic review. Information and Software Technology. 2008; 50(910):833-59.

[27] Connolly A, Kick A. What differentiates early organization adopters of bitcoin from non-adopters? AMCIS 2015.

[28] Rogers EM. Diffusion of innovations. Simon and Schuster; 2010.

[29] Polasik M, Piotrowska AI, Wisniewski TP, Kotkowski R, Lightfoot G. Price fluctuations and the use of bitcoin: an empirical inquiry. International Journal of Electronic Commerce. 2015; 20(1):9-49.

[30] Walton JB, Dhillon G. Understanding digital crime, trust, and control in blockchain technologies. AMCIS 2017.

[31] Janze C, Gvozdevskiy I. What drives the competition of cryptocurrency exchanges? Examining the role of the market and community. ICIS 2017.

[32] Rukanova B, Reuver M, Henningsson S, Nikayin F, Tan YH. Overcoming blockages to collective innovation in digital infrastructures: the case of mobile payment. ECIS 2017. 
[33] Glaser F. Pervasive decentralisation of digital infrastructures: a framework for blockchain enabled system and use case analysis. HICSS 2017.

[34] Grinberg R. Bitcoin: an innovative alternative digital currency. Hastings Sci. \& Tech. LJ. 2012; 4:159.

[35] Kazan E, Damsgaard J. A framework for analyzing digital payment as a multi-sided platform: a study of three european NFC solutions. ECIS 2013.

[36] Gervais A, Karame GO, Wüst K, Glykantzis V, Ritzdorf H, Capkun S. On the security and performance of proof of work blockchains. In proceedings of the ACM SIGSAC conference on computer and communications security 2016 (pp. 316). ACM.

[37] Abramova S, Böhme R. Perceived benefit and risk as multidimensional determinants of bitcoin use: a quantitative exploratory study. ICIS 2016.

[38] Huang DY, Dharmdasani H, Meiklejohn S, Dave V, Grier C, McCoy D, et al. Bitcoin: monetizing stolen cycles. In NDSS 2014.

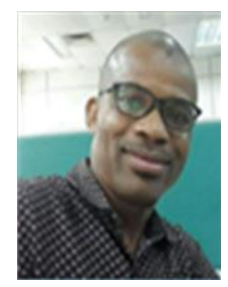

M. SarkinTudu received his Bachelor and Master Degrees in Computer Science from Usmanu Danfodiyo University, Sokoto Nigeria, (UDUS) and Universiti Putra Malaysia (UPM) respectively. $\mathrm{He}$ is currently a $\mathrm{PhD}$ candidate in Information Technology in the School of Computing of Universiti Utara Malaysia (UUM). His research interest is IT project management, Open Source Software (OSS) governance problems with focus on Public Blockchain technology with specific interest in cryptocurrencies with its Distributed Autonomus Organization (DAO).

Email: stjabo@gmail.com

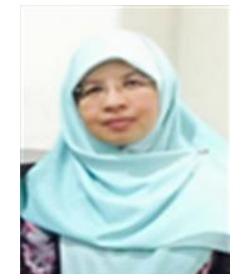

Huda Ibrahim receiveda Masterr in Computer and System Management in 1994 from Creighton University, Omaha, NE, USA and Ph.D (Science and System Management) in 2006 from Universiti Kebangsaan Malaysia. She is currently the Assistant Vice Chancellor of UUM College of Arts and Science and also the Dean of School of Computing (SOC), UUM. She is also a research fellow of the Institute for Advanced and Smart Digital Opportunities (IASDO) of UUM. Her research interests include Information Sharing, ICT Innovation, Smart Cities and Digital Divide Email: huda753@uum.edu.my

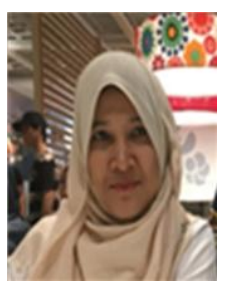

Alawiyah Abd Wahab received her Ph.D in Information Systems from Newcastle University, UK. She is currently a senior lecturer at the School of Computing, Universiti Utara Malaysia. Her research interests mainly lie in the area of e-learning and webbased applications. Her works aim to inform understanding of how the internet and related technologies affect organizations and societies.

Email: alawiyah@uum.edu.my 